\title{
Wierling, Dorothee: Mit Rohkaffee handeln. Hamburger Kaffee-Importeure im 20. Jahrhundert, 384 S., Dölling und Galitz, Hamburg 2018.
}

\author{
Martin Lutz
}

Online publiziert: 20. April 2020

(C) Der/die Autor(en) 2020

Kaffee hat in der jüngsten globalisierungs- und kulturhistorisch inspirierten Wirtschaftsgeschichte vielfach Beachtung gefunden. Das Buch von Dorothee Wierling knüpft an diesen Forschungsdiskurs (Dejung: Die Fäden des globalen Marktes, 2013; Rischbieter: Mikro-Ökonomie der Globalisierung, 2011) mit Fokus auf Hamburger Kaffeeimporteure im 20. Jahrhundert an. Es handelt sich um eine theoretisch reflektierte, empirisch ungemein dichte und anschaulich geschriebene Analyse einer kleinen, gemeinschaftlich organisierten Gruppe von Männern als tragendende Akteure im deutschen und kontinentaleuropäischen Kaffeehandel während des Untersuchungszeitraums. Wierling verbindet auf produktive Weise unterschiedliche historiografische Ansätze, wie beispielsweise aus der Wirtschafts- und Sozialgeschichte, Produktgeschichte, Stadtgeschichte und Globalgeschichte. Sie zeigt darin anhand der bürgerlichen Kaufmannsgruppe den Zusammenhang von Werten, Sozialstruktur und ökonomischem Handeln im Zeitalter ,der Extreme“ (S. 10). Dabei verknüpft sie elegant die Analyse im Kontext des Globalen und Lokalen mit dem zentralen Hamburger Ort Sandtorkai sowie Phasen von Globalisierung und Deglobalisierung in unterschiedlichen politischen Regimen. Zahlreiche Karten, Fotografien und Tabellen veranschaulichen die Studie und legen auch die quantitativen Ergebnisse der Analyse dar.

Das Buch ist übersichtlich gegliedert in einen Teil zu den grundlegenden Strukturen im Kaffeehandel und einen chronologischen Teil. In den beiden Kapiteln des ersten Teils legt die Autorin die Basis für ihren akteurszentrierten Zugriff, indem sie zunächst die Gruppe relevanter Akteure umreißt und ihre Bedeutung im Verein der am Caffeehandel betheiligten Firmen betont. Mit Rückgriff auf Pierre Bourdieus Habitus-Begriff misst sie dem Selbstverständnis einer „,hanseatischen“ Kaufmanns-

M. Lutz $(\bowtie)$

Humboldt-Universität zu Berlin, Berlin, Deutschland

E-Mail: Martin.Lutz@hu-berlin.de 
kultur (S. 19) einen zentralen Stellenwert bei der Formierung von Normen bei. Ehre, Standesbewusstsein, Heiratsstrategien, Vertrauen und die quasi ständische Organisation der Kaufmannsgemeinschaft waren tragende Säulen dieser Kultur, deren Mitglieder sich bei aller Konkurrenz auch als Teil einer „Familie“ (S. 56) identifizierten. Deutlich werden hier die moralischen Wertvorstellungen, sozialen Verbindungen und gemeinschaftlichen Praktiken der Kaufmannsgemeinschaft. Wierling bindet hier auch an die institutionentheoretische Verwendung des Vertrauensbegriffs an. Sie verzichtet allerdings auf eine Verknüpfung mit der aktuellen Diskussion über moral economies.

Der zweite Teil des Buchs beginnt mit einem kurzen Prolog zum 25. Jubiläum des Vereins der am Caffeehandel betheiligten Firmen 1911 und orientiert sich in der folgenden Gliederung an den gängigen Zäsuren deutscher Geschichte im 20. Jahrhundert. Zunächst untersucht Wierling den Einfluss des Ersten Weltkriegs auf die Hamburger Kaffeeimporteure und bindet an das gängige Narrativ einer rückläufigen Globalisierung an. Daneben war für den Kaffeehandel, wie auch für andere Wirtschaftsbranchen, der zunehmende staatliche Einfluss entscheidend. Staat und Hamburger Kaffeeimporteure, so kann Wierling klar zeigen, standen dabei in einem widersprüchlichen Verhältnis zueinander. Einerseits bewerteten die Kaffeeimporteure, deren Geschäft vor 1914 entscheidend durch globalen Freihandel und die unabhängige Organisation im Verein geprägt war, den staatlichen Einfluss sehr kritisch. Anderseits profitierten sie von regulierenden Maßnahmen des Staates, dessen diplomatischer Unterstützung und dadurch verlässlicheren Gewinnerwartungen.

Konfliktlinien und Kooperationsmöglichkeiten boten sich insbesondere im 1916 gebildeten Kriegsausschuss für Kaffee, Tee und deren Ersatzstoffe. Diesen hatten die Hamburger erfolglos zu verhindern versucht und mussten sich in der Ausschussarbeit dann mit der erstarkten Bremer Konkurrenz auseinandersetzen. Während der Kriegszeit profitierten die Kaffeeimporteure einerseits von der, wenn auch im Vergleich mit anderen Nahrungsmitteln untergeordneten, Bedeutung des Kaffees als Konsumgut bei der Versorgung von Militär und Bevölkerung. Andererseits sank das Volumen des Importgeschäfts deutlich. Daher forderten die Hamburger Importeure bereits vor Kriegsende eine ,sofortige Rückkehr zum Freihandel“ im Frieden (S. 90), in der Hoffnung, an die dominierende Rolle beim deutschen Kaffeeimport vor 1914 anknüpfen zu können.

Die Weimarer Republik (Kapitel III) stand für die Hamburger Kaufleute unter diesem prioritären Vorzeichen. Sie wirkten zunächst an der bereits am 21. Oktober 1918 erfolgten Gründung des privatwirtschaftlichen Kaffee-Einfuhr-Vereins (KEV) mit, in dem sich Importeure, Großhändler und Röster zusammenschlossen. Allerdings hatte dieser seit der Freigabe von Rohkaffee-Importen im Mai 1921 nur eine untergeordnete Bedeutung inne und wurde fünf Monate später wieder aufgelöst. Als nach Hyperinflation und Dawes-Plan die schrittweise Re-Integration in den Weltmarkt gelang, mussten sich die Hamburger Importeure dann an die strukturellen Veränderungen des Kaffeemarkts in der vergangenen Dekade anpassen. In Kapitel V zeichnet Wierling minutiös die Anpassung und Konfliktlinien der Hamburger Importeure im Nationalsozialismus nach. Bedeutsam ist neben dem Umgang mit jüdischen Mitgliedern in den eigenen Reihen auch der Versuch, den Verein als privatwirtschaftliche Selbstorganisation vor dem versuchten staatlichen Zugriff zu schüt- 
zen, was den Kaufleuten zumindest zeitweise und teilweise auch gelang. Weitaus stärker als der Erste Weltkrieg bildete der Zweite Weltkrieg einen tiefen Einschnitt für den Hamburger Rohkaffeehandel als Importhafen und als sozialer Bezugspunkt der Kaufmannsgemeinschaft (Kapitel VI). Manche deren Mitglieder wurden unmittelbar in die Nahrungsmittelpolitik des NS insbesondere in der Ukraine eingebunden und dabei beispielsweise auch Zeugen des Massenmords an der jüdischen Bevölkerung in Riwne (S. 199). In der unmittelbaren Nachkriegszeit trat der Verein der am Caffeehandel betheiligten Firmen bereits im Mai 1945 zusammen, um - laut Protokoll ganz nüchtern - schnellstmöglich an die Geschäftstätigkeit der Vorkriegszeit anzuknüpfen. Geprägt war der „Import“ freilich zunächst auch durch Schmuggel. Die Rückkehr zum Freihandel gelang erst 1955, als Importbeschränkungen weitgehend entfielen. Dabei macht Wierling deutlich, dass das Nachkriegsgeschäft stark unter den Bedingungen des Kalten Kriegs litt, der für die Hamburger Kaffeeimporteure den Verlust des ostdeutschen und -europäischen „Hafenhinterlandes“ (S. 258) bedeutete. Über die Leipziger Messe konnten sie zwar weiterhin am Export in die DDR und in osteuropäische Länder partizipieren, doch die europäische Integration im Rahmen der Europäischen Wirtschaftsgemeinschaft war ungleich bedeutender, wie auch das internationale Kaffeeabkommen von 1963. Der bundesrepublikanische Strukturwandel (Kapitel IX) äußerte sich bei den Hamburger Kaffeeimporteuren unter anderem in Konkursen und in der Konzentration von Unternehmen in der Kaffeebranche, wie Wierling anschaulich an zwei Familienfirmen zeigt. Im abschließenden Kapitel X zieht die Autorin eine Bilanz. Sie betont die Wichtigkeit der intergenerationalen, familiengeprägten Struktur vieler Hamburger Kaffeeimporteure. Deren Gemeinschaft, basierend auf dem Wertesystem hanseatischer Kaufmannskultur, habe durch die Nachkriegsbesatzung ihren ,eigentlichen historischen Bruch“ erlebt und die Sozialstruktur von Grund auf verändert. Aus einer ständisch orientierten Gruppierung wurde eine „Händler-Klasse“, in der nun weniger das Gemeinschaftsgefühl als Wettbewerb um Marktanteile galt (S. 333). Mit einem kurzen Ausblick auf die Fair-Trade-Bewegung und die Auswirkungen des Klimawandels auf das Kaffeegeschäft schließt die Untersuchung und bindet damit an aktuelle Diskussionen an.

Wierlings Buch ist auch bemerkenswert, als der empirische Zugriff auf den Gegenstand nicht leicht zu bewältigen war. Archive der meist familiengeführten Unternehmen im Kaffeehandel sind nur in Ausnahmefällen erhalten. Daher erwies sich der Fokus auf den Verein der am Caffeehandel betheiligten Firmen als wichtige Mittlerorganisation als ungemein produktiv, auch wenn dadurch der akteurszentrierte Ansatz etwas eingeschränkt ist. Wierling ergänzt die Quellengrundlage durch eine beeindruckende Heranziehung von Dokumenten unter anderem aus dem Bundesarchiv Berlin und Koblenz, dem Archiv der Handelskammer Hamburg und dem Politischen Archiv des Auswärtigen Amts. Auch Oral-History-Interviews zieht sie heran. Das auch in der überregionalen Tagespresse mehrfach besprochene Buch liefert einen wertvollen Beitrag zur Lokalgeschichte des Handelsplatzes Hamburg, der deutschen Wirtschafts-, Sozial- und Kulturgeschichte sowie zur Globalisierungsgeschichte.

Funding Open Access funding provided by Projekt DEAL. 
Open Access Dieser Artikel wird unter der Creative Commons Namensnennung 4.0 International Lizenz veröffentlicht, welche die Nutzung, Vervielfältigung, Bearbeitung, Verbreitung und Wiedergabe in jeglichem Medium und Format erlaubt, sofern Sie den/die ursprünglichen Autor(en) und die Quelle ordnungsgemäß nennen, einen Link zur Creative Commons Lizenz beifügen und angeben, ob Änderungen vorgenommen wurden.

Die in diesem Artikel enthaltenen Bilder und sonstiges Drittmaterial unterliegen ebenfalls der genannten Creative Commons Lizenz, sofern sich aus der Abbildungslegende nichts anderes ergibt. Sofern das betreffende Material nicht unter der genannten Creative Commons Lizenz steht und die betreffende Handlung nicht nach gesetzlichen Vorschriften erlaubt ist, ist für die oben aufgeführten Weiterverwendungen des Materials die Einwilligung des jeweiligen Rechteinhabers einzuholen.

Weitere Details zur Lizenz entnehmen Sie bitte der Lizenzinformation auf http://creativecommons.org/ licenses/by/4.0/deed.de.

\title{
Slobodian, Quinn: Globalists. The End of Empire and the Birth of Neoliberalism, 400 S., Harvard UP, Cambridge, MA/London 2018.
}

\author{
Alexander Leipold \\ Online publiziert: 27. März 2020 \\ (c) Gesellschaft zur wissenschaftlichen Förderung politischer Literatur e.V. and the Author(s) 2020
}

In den Sozialwissenschaften gilt der Begriff des Neoliberalismus bisweilen als leerer Signifikant, der mal für eine Politik der Privatisierung und mal als Beispiel für Konzernlobbyismus dient. Entgegen dieser verkürzten Lesart richtet Quinn Slobodian, Historiker am Wellesley College, die vorliegende Studie, die auf der Auswertung von Quellenmaterial aus acht Archiven und einer breiten Basis an Sekundärliteratur gründet. Als „Globalisten“ versteht der Autor eine Gruppe von Intellektuellen, die nach Ende des Ersten Weltkrieges Überlegungen zur Sicherung des Privateigentums vor demokratischer Mitbestimmung anstellten. Mit seiner Arbeit legt Slobodian ein notwendiges Korrektiv der Forschung zum Neoliberalismus vor und verknüpft bisher nebeneinanderliegende Forschungsstränge aus Politischer Soziologie und Geschichte. Das Buch richtet sich aufgrund seiner Anlage an Interessierte aus den Feldern der Wissenssoziologie, der Internationalen Beziehungen, aber auch der Demokratieforschung. Sein eleganter Schreibstil, die Erschließung des Bandes durch

\footnotetext{
A. Leipold $(\bowtie)$

Leuphana Universität Lüneburg, Lüneburg, Deutschland

E-Mail: alexander.leipold@leuphana.de
} 